\title{
Synthesis and Optical Properties of Polystyrene Doped with DCM Thin Films
}

\author{
Akeel Shaker Tuhaiwer \\ Physics Department, College of Science, AL Muthanna University. \\ ${ }^{*}$ Corresponding Author: Akeelshaker2000@gmail.com
}

Received:25/12/2017, Accepted:16/01/2018, Published:26/3/2018.

DOI: $10.52113 / 2 / 05.01 .2018 / 8-18$

\begin{abstract}
In the present work Polystyrene (PS) polymer doped with DCM laser dye thin films have been prepared on glass substrate using casting method. The final samples of PS doped with DCM thin films were treated with different annealing temperatures at $(30,40,50,60){ }^{\circ} \mathrm{C}$. Optical absorption and transmission spectra of the prepared thin films were recorded using UV-VIS double beam spectrometer in the wavelength range from 190 to $1100 \mathrm{~nm}$. The results shown that the allowed direct electronic transitions energy gap increased from 2.08 up to $2.25 \mathrm{e}$. V as the temperature increasing from 30 to $60^{\circ} \mathrm{C}$, whereas the allowed indirect electronic transition energy gap increase from 2.02 up to $2.17 \mathrm{eV}$ as the temperature increase from 30 to $60^{\circ} \mathrm{C}$. The effect of temperature on the optical properties such as Urbach's energy, Refractive index, Extinction coefficient, Critical angle, Brewster angle, and Finesse coefficient of PS doped with DCM thin films also studied in this work.
\end{abstract}

C2018 Al Muthanna University. All rights reserved

Keywords: Optical properties, PS, DCM, thin films, cast method.

\section{Introduction}

Studying thin films is one of the most suitable means to know many of their physical properties, which are difficult to obtain in their natural. [1] The development in the field of thin films has led to a variety of research to study the physical properties of these thin films, and to prepare thin films with a high specification of purity and accuracy and control the thickness of the thin films and homogeneous requires systems and devices accurate and complex, which require high costs. [2,3] Owing to the properties of thin films distinctive and large importance, it has multiplied thier uses and practical applications and industry in various fields. [4] They are currently used in the construction of electronic components of complex devices, depositing their layers one by one. [5] It is also of great importance in the glass industry, ceramic industry and the glass coating by 
silver salts and in manufacturing solar cells, capacitors, filters, transistors, electric switches, reagents and in countless fields $[6,7]$ Polymeric material has various characteristics which can be controlled and monitored. These characteristics are including light-weight, mechanical flexibility, high-dielectric strength, fracture tolerance, high chemical resistance, easy processibility, and low manufacturing cost. Moreover they can be configured into almost any conceivable shape and their properties can be tailored to suit many applications.[8] Polymer thin films find wide range of technological applications such as coatings, adhesives, lithography, sensors and as solid state electrochemical cells they can be directly deposited on to chips in any shape or size.[9]. Polystyrene is a synthetic aromic polymer that is made from the monomer styrene, which is a liquid petrochemical. Typically it can be heated and melted for molding or extrusion purposes; eventually it will become a solid again once it has cooled off. Polystyrene can be transparent or have multiple different colors on it. Some examples that polystyrene is used for are: disposable cutlery, plastic models, CD's \& DVD cases, and smoke detector housings.

[10-11]
Polystyrene belongs to the group of standard thermoplastics that also includes polyethylene, polypropylene, and polyvinyl chloride. Because of its special properties polystyrene can be used in an extremely wide range of applications. Polystyrene an amorphous, optically clear thermoplastic material, which is flexible as thin films, is chosen as the host matrix because of its ideal properties for investigating the optical properties of the DCM laser dye. (PS) is in solid (glassy) state at room temperature but flows if heated above its glass transition temperature and becomes solid again when cooled. Introduction of DCM laser dye into polymeric matrices can in general, modify the optical, electrical and mechanical properties of the polymer. [12-13] In current work, PS was mixed with DCM laser dye to obtain homogenous thin films and study the optical properties of these films.

The absorption coefficient ( $\alpha)$ was calculated using the equation [14]:

$\alpha=\frac{2.303 A}{d}$

Where: $d$ is the film thickness.

The optical absorption coefficient is very important because it supplies information about the electronic transition using the equation [15]: 


$$
\alpha=\frac{\left(h v-E_{g}\right)^{\frac{1}{2}}}{h v}
$$

Where $B$ is constant

$h v$ is photon energy and $E_{g}$ is energy gap.

The optical absorption and especially the absorption band edge is useful way for studying optically prompted transition and provides information about the configuration and optical energy gap in thin films. The absorption edge in many materials follows the Urbach rule [16]:

$\alpha=\alpha_{o} \operatorname{Exp}\left(\frac{h v}{E_{u}}\right)$

Where $\alpha_{o}$ is constant

$E_{u}$ is urbach energy

The extinction coefficient $(K)$ which obtained in terms of the absorption coefficient is given by the equation [17]:

$$
K=\frac{\lambda \alpha}{4 \pi}
$$

The refractive index $(n)$ can be calculated by using equation [18]:

$$
n=\frac{1+\sqrt{R}}{1-\sqrt{R}}
$$

Where $R$ is reflectivity

In order to analyze the refractive index dispersion of PS doped with DCM thin films at various annealing temperatures used the single-oscillator model developed by DiDomenico and Wemple. The refractive index can be expressed in terms of the dispersion energy $E_{d}$ and single- oscillator energy $E_{o}$. The single-oscillator model for the refractive index dispersion is expressed in equation [19]:

$n^{2}=1+\frac{E_{d} E_{o}}{E_{o}^{2}-(h v)^{2}}$

Fineness coefficient $(F)$ was investigated by calculating the reflectance, the equation of fineness coefficient is given by [20]:

$F=\frac{4 R}{(1-R)^{2}}$

As for the critical angle $\left(\theta_{c}\right)$ was calculated by measuring the refractive index which is given by the equation [21]:

$\theta_{c}=\sin ^{-1}\left(\frac{1}{n}\right)$

The brewster angle $\left(\theta_{B}\right)$ was calculated by measuring the refractive index which is resulted from the equation [22]:

$\theta_{B}=\tan ^{-1}(n)$

\section{Materials and Methods (Experimental} Procedure)

To prepare PS doped with DCM thin films, firstly dissolving $0.015 \mathrm{gm}$ from $\mathrm{DCM}$ in $10 \mathrm{ml}$ tetrahydrofuran (THF) solvent and stirrer about 30 minutes to obtain homogenous solution, then 2gm PS dissolve in $30 \mathrm{ml}$ THF with 2 hours vigorous stirring to get the polymer solution. To synthesis the PS-DCM thin films, 2ml DCM solution mixed with $5 \mathrm{ml}$ PS solution and casting on glass substrate at room temperature. To study the effect of temperature on the optical properties of 
films, applied this effect to the final samples as $30,40,50$ and $60{ }^{\circ} \mathrm{C}$.

Thin films thickness was measured using the optical interferometer method employing He-Ne laser $0.632 \mu \mathrm{m}$, and found to be $0.47 \mu \mathrm{m}$ for all samples. The optical absorption and transmission spectra of DCM doped with PS thin films were recorded using UV-VIS double beam spectrometer in the wavelength range from 190 to $1100 \mathrm{~nm}$.

\section{Results and Discussion}

The optical properties measurements for PS doped with DCM thin films at different temperatures were obtained using UV-VIS spectrophotometer on glass substrate. The absorbance of these films depends on the annealing temperature as a function of wavelength is shown in figure (1).

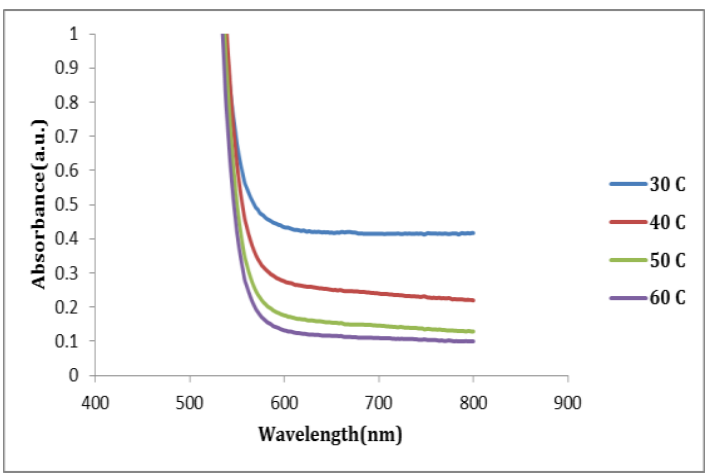

Fig.1: Absorption spectra of PS doped with DCM thin films at different annealing temperatures.

From figure (1) It can observe that the absorption of all films decreases with the annealing temperature increasing, that is due to the shift in the absorption edge obtain from the heat treatment is explained by a change in the defect structure of the films. [23]

Figure (2) shows the absorption coefficient for PS doped with DCM thin films at different annealing temperatures determined by using Eq.(1). The absorption coefficient of these films decreases with the increasing annealing temperature, because of the dependency of absorption coefficient on the films absorption to the incident light.

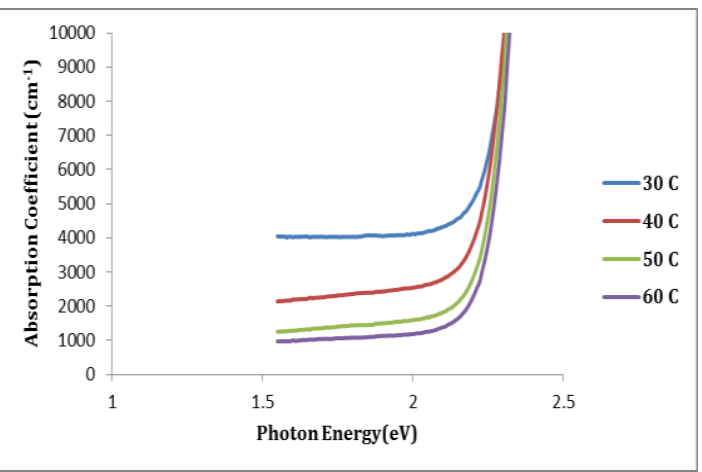

Fig.2: Absorption coefficient spectra of PS doped with DCM thin films at different annealing temperatures.

The energy gap can be calculated from Eq.(2). The relations drawn between $(\alpha h v)^{2}$, $(\alpha h v)^{1 / 2}$ and photon energy (hv), as shown in Fig. (3) and (4) which illustrates allowed direct electronic transition and Fig. (4) illustrates allowed indirect electronic transition. The energy gap value depends on the films deposition conditions and its preparation method [24]. From table (1), one can be observed that energy gap of direct and indirect transitions values $\left(\mathrm{E}_{\mathrm{g}}\right)$ is 
increasing with the increasing of annealing temperature for all films. Where the minimum value of the allowed direct electronic transition energy gap was (2.08) at $30{ }^{\circ} \mathrm{C}$ and the highest was $(2.25)$ at $60{ }^{\circ} \mathrm{C}$, while the minimum value of the allowed indirect electronic transition energy gap was (2.02) at $30{ }^{\circ} \mathrm{C}$ and the highest was (2.17) at $60{ }^{\circ} \mathrm{C}$.

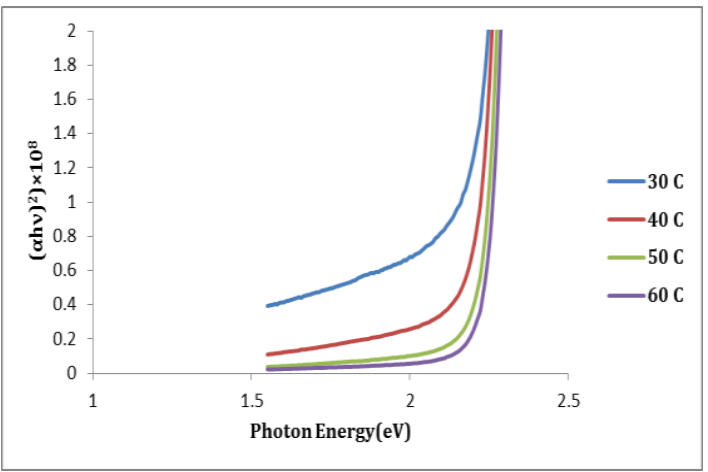

Fig.3: Relationship between $(\alpha h v)^{2}$ and photon energy (e.V) for PS-DCM the films at different annealing temperatures.

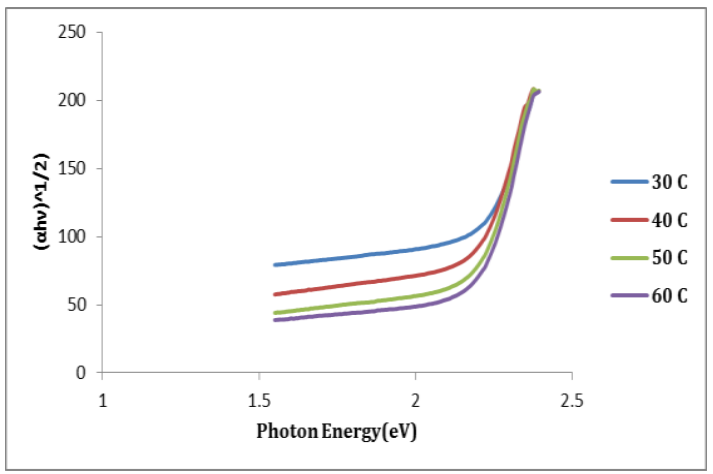

Fig.4: Relationship between $(\alpha h v)^{1 / 2}$ and photon energy (e.V) for PS-DCM the films at different annealing temperatures.

The direct and indirect electronics transitions energy gaps for prepared thin films at different annealing temperatures are summarized in table 1, which discusses the increase and decrease in $E_{g}$.

Figure (5) shows the dependence of the first derivative of the absorption on the photon energy for prepared thin films at different annealing temperatures.

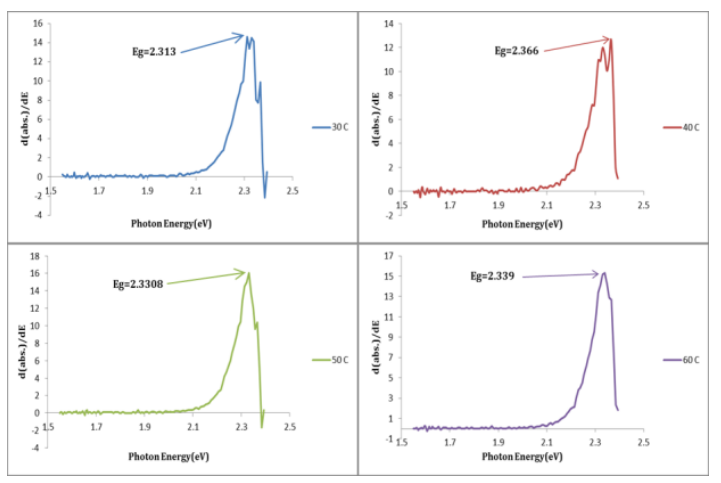

Fig.5: Relationship between the first derivative of the absorption and the photon energy for PS doped with DCM thin films at different annealing temperatures.

When taking the first derivative of the absorption as a function of the photon energy, it was observed that the increase in annealing temperature has led to increase in the values of the energy gap represented by the curve for all samples as in Fig.5, the energy gap values which obtained from the first derivative are recorded in the table 1, this is inverse the results of Tauc's model of the energy band gap values.

The values of Urbach energy were calculated as the reflexive incline of the linear portion of the plot. Moreover, Fig.6, shows the plot of $(\ln \alpha)$ versus photon 
energy $(\mathrm{eV})$ for PS doped with DCM thin films at different annealing temperatures and the values of Urbach energy are summarized in table 1 .

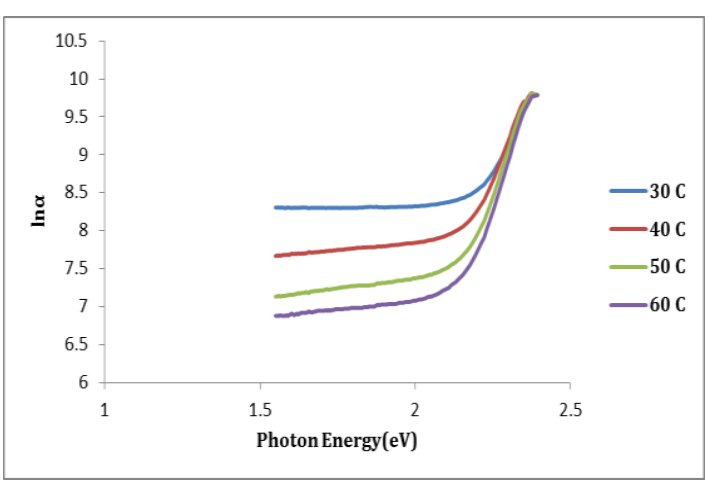

Fig.6: Relationship between Urbach energy and photon energy (e.V) for PS-DCM the films at different annealing temperatures.

One can observe from Fig.6, that the Urbach energy values were decreased with the increasing of energy gap and with the increasing of annealing temperatures, this behavior due to the decreasing of defect levels in the allowed band gap by increasing annealing temperatures so these results an agree with ref. [25].

The spectral dependence of the extinction coefficient $(\mathrm{K})$ on the wavelength variation for PS doped with DCM thin films at different annealing temperatures was obtained using Eq.(4), this relation is shown in Fig. 7.

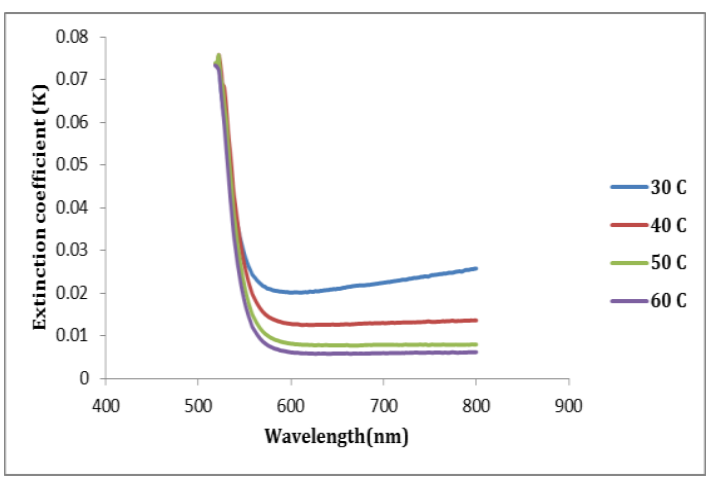

Fig.7: Relationship between extinction coefficient and wavelength for PS-DCM the films at different annealing temperatures.

It can be noticed that the extinction coefficient had lowering values at low temperatures. Extinction coefficient behaves in the same behavior of absorption spectrum since they are joined mathematically.

The refractive indices for PS doped with DCM thin films at different annealing temperatures were determined from Eq.(5). Figure (8) shows the variation in refractive index of these films in the wavelength range of (400-900nm). The increasing in the annealing temperature results an increasing in the refractive index in the visible/ near infrared region, but the refractive index tends to be unaffected at the UV region. 


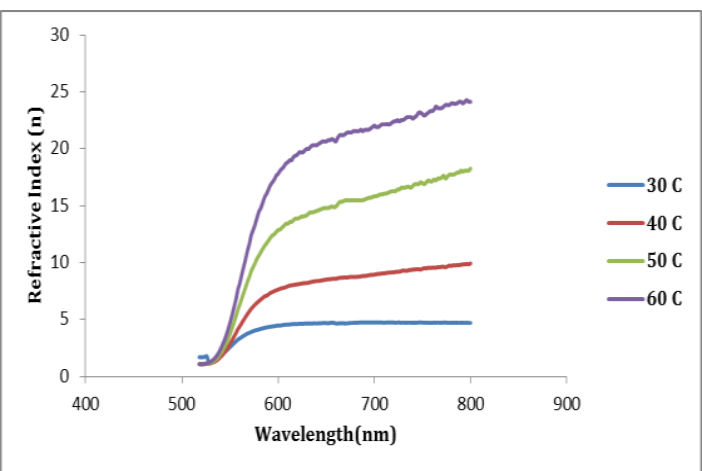

Fig.8: Refractive index for PS-DCM the films at different annealing temperatures.

From Fig. 8 it is observed an increasing in the refractive index this due to increased temperature effect for all prepared films [ 26].

The relation $1 /\left(\mathrm{n}^{2}-1\right)$ versus $h v^{2}$ is plotted in Fig.9, that it allows us to determine the oscillator parameters $E_{o}$ and $E_{d}$ values. $E_{o}$ is an average energy gap and can be related to the band gap, $\mathrm{E}_{\mathrm{g}}$ in close approximation $\mathrm{E}_{\mathrm{o}} \approx 2 \mathrm{E}_{\mathrm{g}}[27]$.

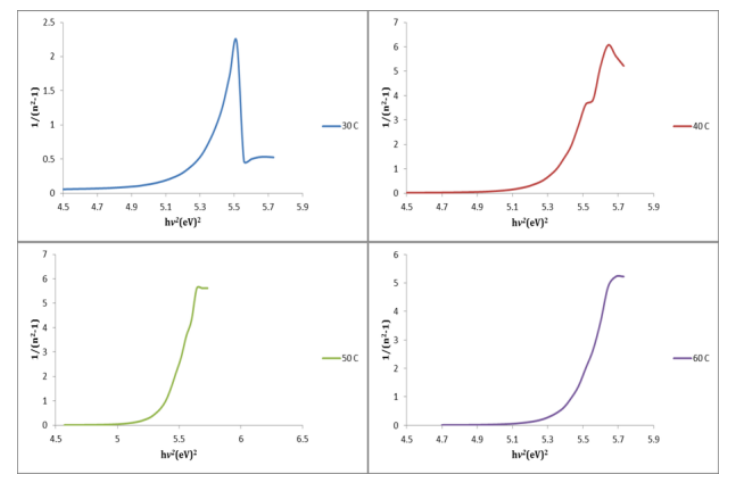

Fig.9: Relationship between $1 /\left(n^{2}-1\right)$ and $\mathrm{hv}^{2}$ of PS doped with DCM thin films at different annealing temperatures.

The values of $E_{o}$ and $E_{d}$ were calculated from the slope and intercept on the vertical axis of $1 /\left(n^{2}-1\right)$ versus $h v^{2}$ plot, which listed in table 1 .

The coefficients of finesse of PS doped with DCM thin films at different annealing temperatures were calculated using Eq.(7). Figure (10), shows that finesse coefficient of PS doped with DCM thin films at different annealing temperatures have the same behavior to their reflectance, since this coefficient depends on reflectance, from another aspect the influences of increasing of annealing temperatures lead to increase the values of finesse coefficient, this is due to the increasing of reflected light as a result of increasing the density after increasing of annealing temperatures, these results agree with ref [28].

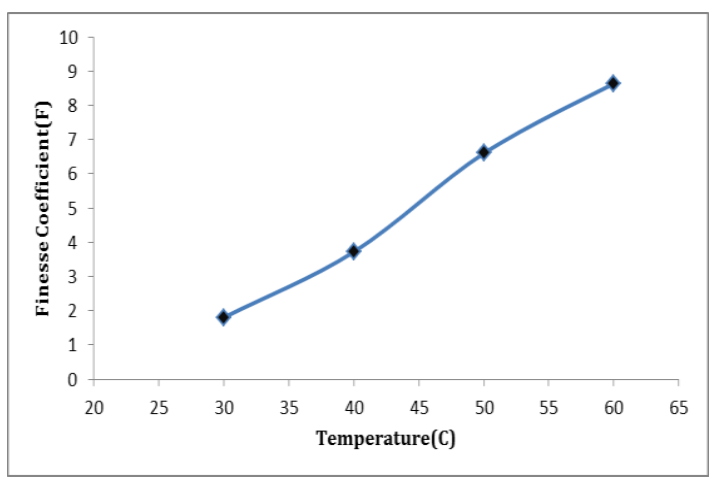

Fig.10: Finesse coefficient for PS-DCM the films at different annealing temperatures.

Figure 11, shows the critical angle of an inverse relationship with the refractive index, any increase in the refractive index causes a decrease in the critical angle, or it is a clear that as annealing temperatures 
increase, the density also increase therefore the incident rays will bend toward normal and cause a critical angle to be decrease[28].

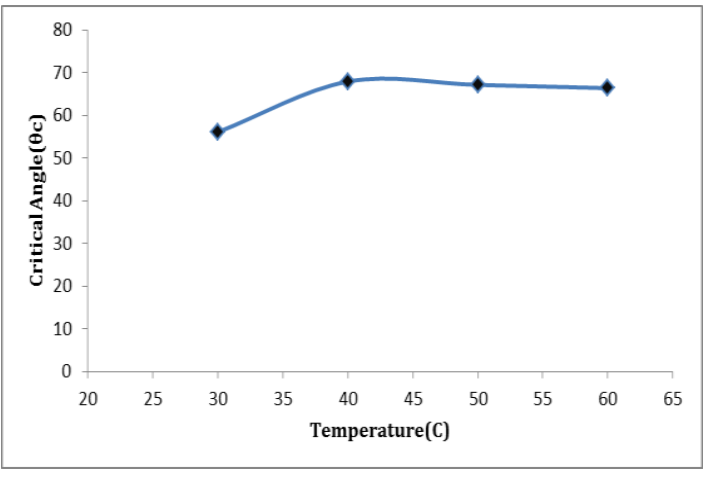

Fig.11: Critical angle for PS-DCM the films at different annealing temperatures.

The values of Brewster angle depend mainly on the values of the refractive index relationship with the refractive index and Fig.12 shows that the Brewster angle increases with increasing annealing and the reason for this is due to the increasing of refractive index with increased annealing temperatures.

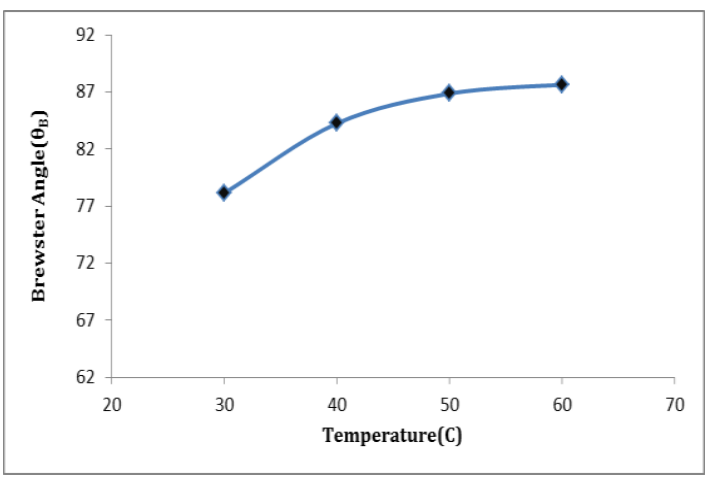

Fig.12: Brewster for PS-DCM the films at different annealing temperatures.

\section{Conclusion}

Polystyrene and DCM doped with polystyrene thin films were prepared casting method was used in this work and the effect of the annealing temperature on the optical properties was studied using UV-VIS spectrometer. Increasing of the annealing temperature for all films causes an increasing in the direct electronic transitions were (2.08-2.25)eV, and indirect electronic transitions were found to be (2.02-2.17)eV. The energy gab from derivative absorption spectra (2.3132.339)eV. This increasing also results an increase in the refractive index, whereas, it causes decreasing in extinction coefficient for all films. Urbach tail assures that the crosslink is decreased as the annealing temperatures increase (0.967-0.411) eV. 
MJPS, VOL.(5), NO.(1), 2018

Table 1: The calculated parameters for PS doped with DCM thin films at different temperatures

\begin{tabular}{|c|c|c|c|c|c|c|}
\hline $\begin{array}{c}\text { Temperatures } \\
{ }^{\circ} \mathrm{C}\end{array}$ & $\begin{array}{c}\text { single- } \\
\text { oscillator } \\
\text { energy } \mathrm{E}_{\mathrm{o}}(\mathrm{eV})\end{array}$ & $\begin{array}{c}\text { Dispersion } \\
\text { energy } \\
\mathrm{E}_{\mathrm{d}}(\mathrm{eV})\end{array}$ & $\begin{array}{c}\text { Urbach's } \\
\text { energy } \\
\mathrm{E}_{\mathrm{u}}(\mathrm{eV})\end{array}$ & $\begin{array}{c}\mathrm{E}_{\mathrm{g}} \text { Direct }(\mathrm{eV}), \\
\text { from Tauc } \\
\text { model }\end{array}$ & $\begin{array}{c}\mathrm{E}_{\mathrm{g}} \text { Indirect }(\mathrm{eV}), \\
\text { from Tauc } \\
\text { model }\end{array}$ & $\begin{array}{c}\mathrm{E}_{\mathrm{g}}(\mathrm{eV}) \text {, from } \\
\text { derivative abs. } \\
\text { spectra }\end{array}$ \\
\hline 30 & 3.987 & 4.819 & 0.967 & 2.08 & 2.02 & 2.313 \\
\hline 40 & 4.057 & 4.881 & 0.5602 & 2.14 & 2.08 & 2.366 \\
\hline 50 & 4.23404 & 4.9517 & 0.437 & 2.2 & 2.14 & 2.3308 \\
\hline 60 & 4.348 & 5.041 & 0.411 & 2.25 & 2.17 & 2.339 \\
\hline
\end{tabular}

\section{References}

[1] Heavens. O. S., (1970) "The Film physics", Methen Young Co. Ltd.

[2] Ueda. R and Millin. J. B., (1975) "Crystal Growth and Characterization", Mc Graw- Hill.

[3] Chopra. K. L., (1985), "Thin Film Phenomena 9", Mc. Graw- Hill, New York.

[4] Macleod. H.A., (2001), "Thin Film Optical Fillers",Academic Press, 3rd ed.

[5] Oaring. M.,(1977), "The Materials Science of Thin Films", Academic Press.

[6] Eckertova. L.,(1977), "Physics of Thin Films", Plenum presses, New York and London.

[7] Son. J.R.,(1986), "Thin Films Technologies", Mc Graw-Hill, 2nd ed.
[8] Hashim. A. A. (2010),"Polymer dipoles relaxation and potential energy (New Simulation Model)", Polymer Thin Films

[9] Kumar. K. N. and Buddhudu. S.,(2014),"Enhanced

Photoluminescence of $\mathrm{Mn}^{2+} \mathrm{Tb}^{3+}$ Ions Doped PEO+PVP Blended Polymer Films", Proc Indian Natn Sci Acad , 80, 345-354.

[10] Arakawa. E. T., Hamm. R. N. and Williams. M. W., (1977), "Optical Properties of Polystyrene from the near-infrared to the $\mathrm{x}$-ray region and convergence of optical sum rules", Physical Review B, 15, 3243-3253.

[11] Jaleh. B., Madad. M. S., Tabrizi. M. F., Habibi. S.,Golbedaghi. R. and Keymanesh. M.R.,(2011), " UVDegradation Effect on Optical and Surface Properties of Polystyrene$\mathrm{TiO}_{2}$ Nanocomposite Film", J. Iran. Chem. Soc., 8, S161-S168. 
[12] Sharp J. S., Teichroeb J. H.,Forrest J. A.,(2004), "The properties of free polymer surfaces and their influence on the glass transition temperature of thin polystyrene films", The European Physical Journal E, $15,473-487$

[13] Ahmed Namah Mohamed "Studying the optical properties of thin films prepared from polystyrene doped with DCM dye". 61(2015), 176-181.

[14] Akeel Shaker Tuhaiwer, (2016), " Optical study on deposed polystyrene doped DCM laser dye thin films at different annealing temperatures" International Letters of Chemistry, Physics and Astronomy, 68, 39-47.

[15] Nadir Fadhil Habubi, Bashar Hatem Abed, Sami Salman Chiad, (2012), "Optical properties of $\mathrm{BaCl}_{2}$ doped poly (vinyl alcohol) films" Iraqi Journal of Physics, 10, 18-22.

[16] Nadir Fadhil Habubi, Reem Saadi Khaleel and Sami Salman Chiad, (2010), "Influence of Co Doping on the Optical Properties of $\mathrm{ZnO}$ Thin Films" AL- Mustansiriya J. Sci, 21, 41-47.

[17] Ahmed Namah Mohamed, Akeel Shaker Tuhaiwer, Zaid Saud
Razzaq, (2016), "Optical Properties of Polyvinyl Chloride Doped with DCM dye Thin Films", World Scientific News 30, 45-56.

[18] Rawaa A. Faris and Mohamed K. Dhahir, (2016), “Synthesis Characterization and Optical Properties of Nanostructured Zinc Sulfide Thin Films Obtained by Spray Pyrolysis Deposition" Iraqi J. Laser, Part A, 15, 9-12.

[19] Nadir F.Habubi1, Nabeel A. Bakr, Sabah A. Salman, (2013), “Optical parameters of amorphous selenium deposited by thermal evaporation technique" PCAIJ, 8(2), 54-58.

[20] Abdul Amir Khalaf, (2014), "Effect of Increasing The Concentration Of $\mathrm{Al}\left(\mathrm{SO}_{4}\right)_{2} .12 \mathrm{H}_{2} \mathrm{O}$ On Some Optical Properties Of The Solutions Prepared" Advances in Physics Theories and Applications, 30, 8-14.

[21] Youkghoo kim, Wan Hee Goh ,Taihyun Chang , M.Ree , (2004), "Advance Engineering Materials “, $6,1-2$.

[22] Malgosia Kaczmarek, Yasuo Tomita, (2009), " JOURNAL OF OPTICS A: Pure Appl.”, 11.

[23] Yoshino. K., Nakajima. S., Gu. H. B., and Sugimoto. R., (1987)," Absorption and 
Emission Spectral Changes in a

Poly(3-Alkylthiophene) Solution

with Solvent and Temperature",

Japanese Journal of Applied

Physics, 26

[24] Brodky, M., (1979), "Amorphous

Semiconductors" Sepringer- Verlag,

Berlin, Heidelberg.

[25] Fox, M., (2010), “Optical Properties

of Solid. Great Britain";Oxford

University; New York. USA.

[26] Brodky, M., (1979), "Amorphous

Semiconductors" Sepringer- Verlag,

Berlin, Heidelberg.

[27] Pietro-Alcon.R, Gonzalez-Leal. J.M., Jimenez- Garay R., Marquez E., (2001), "On the Changes in the Structural and Optical Properties Accompanying the Athermal Photovitrification Phenomenon in As50Se50 Thin Film", J.Optoelectron.Adv.Mater., 3, 287

[28] Herman. F.,(1971), "Encyclopedia of Polymer Science and Technology",14, John Wiley and Sons Int., New York. 